\title{
ESCOLAS DE GOVERNO E SEU PAPEL NO APERFEIÇOAMENTO DO DESEMPENHO DOS SERVIDORES PÚBLICOS
}

\section{SCHOOLS OF GOVERNMENT AND THEIR ROLE IN IMPROVING THE PERFORMANCE OF PUBLIC SERVERS}

\author{
Adriano Mello de Andrade ${ }^{1}$
}

Submetido em: 18/05/2021

Aprovado em: 08/06/2021

e25350

\section{RESUMO}

As escolas de governo são instituições que tem por objetivo fornecer capacitação para os servidores públicos, bem como o de profissionalização para cargos de carreira. Nesse interim, o presente artigo tem como objetivo dissertar sobre o papel das escolas de governo no aperfeiçoamento e capacitação de servidores públicos. Para tanto, realizou-se uma pesquisa bibliográfica, de natureza qualitativa e para fins descritivos, utilizando de autores encontrados por meio de buscas em bancos de dados digitais de publicações científicas, principalmente o Google Acadêmico e SciELO (Scientific Eletronic Library Online). Destaca-se as contribuições de Andrade (2012), Pacheco (2002), Fernandes (2015) e Aires et al. (2014) para o desenvolvimento deste artigo, que evidenciaram a importância das escolas de governo, atuando como ferramentas de aperfeiçoamento do funcionalismo público, ao promover a capacitação e o conhecimento desses profissionais. Assim, é possível concluir que as escolas de governo têm um papel fundamental no aperfeiçoamento do desempenho de servidores públicos, o que se reflete na melhoria dos serviços prestados à população e, consequentemente, aumenta da qualidade da Administração Pública. Sendo assim, pode-se concluir que as escolas de governo se configuram como um meio de corroborar para a formação dos servidores públicos, de maneira íntegra, objetivando a construção de sujeitos ativos e conscientes do seu papel na Administração Pública.

PALAVRAS-CHAVE: Escolas de Governo. Papel. Desempenho. Servidores Públicos.

\begin{abstract}
Government schools are institutions that aim to provide training for public servants, as well as professional training for career positions. In the meantime, this article aims to discuss the role of government schools in the improvement and training of public servants. Therefore, a bibliographical research was carried out, qualitative in nature and for descriptive purposes, using authors found through searches in digital databases of scientific publications, mainly Academic Google and SciELO (Scientific Electronic Library Online). The contributions of Andrade (2012), Pacheco (2002), Fernandes (2015) and Aires et al. (2014). For the development of this article, which highlighted the importance of schools of government, acting as tools for the improvement of civil servants, by promoting the training and knowledge of these professionals. Thus, it is possible to conclude that government schools have a fundamental role in improving the performance of public servants, which is reflected in the improvement of services provided to the population and, consequently, increases in the quality of Public Administration. Thus, it can be concluded that government schools are configured as a means to support the training of public servants, in an integral way, aiming at the construction of active subjects aware of their role in Public Administration.
\end{abstract}

KEYWORDS: Schools of Government. Paper. Performance. Public Servants.

1 Mestrando em Ciências Contábeis pela FUNDAÇÃO INSTITUTO CAPIXABA DE PESQUISAS EM CONTABILIDADE, ECONOMIA E FINANÇAS-FUCAPE, possui graduação em Gestão Pública pela Universidade Estácio de Sá, Bacharelado em Ciências Contábeis pela Universidade Estácio de Sá. Graduado em Gestão Pública pela Universidade Estácio de Sá. Pós-graduando em Docência no Ensino Superior pela Universidade Candido Mendes - UCAM. E-mail: adriano.ilha@gmail.com 


\section{RECIMA21 - REVISTA CIENTÍFICA MULTIDISCIPLINAR ISSN 2675-6218}

ESCOLAS DE GOVERNO E SEU PAPEL NO APERFEICOAMENTO DO DESEMPENHO DOS SERVIDORES PÚBLICOS Adriano Mello de Andrade

\section{INTRODUÇÃO}

O presente estudo tem como tema as escolas de governo e seu papel no aperfeiçoamento do desempenho dos servidores públicos, trazendo uma discussão sobre o conceito dessas instituições e de que forma atuam na capacitação do funcionalismo público, dada a necessidade de constante evolução das instituições governamentais, bem como a melhoria da qualidade do serviço prestado à população.

Sendo assim, apresenta-se a seguinte questão norteadora do presente artigo: Qual é a função das escolas de governo e de que forma atua no aperfeiçoamento do desempenho dos servidores públicos?

O desempenho de um país não depende apenas de planejamento direcionados para a solução de seus problemas; ele também precisa de um sistema administrativo competente e capacitado em ciências do governo, nas políticas públicas e nos problemas do setor público, fomentado uma ação assertiva em prol da melhoria do desempenho da máquina estatal.

Nesse interim, surgem as escolas de governo, que de acordo com Fonseca et al. (2015, p. 17):

[...] desempenham papel fundamental para o provimento das competências necessárias ao aperfeiçoamento das organizações públicas, constituem a infraestrutura especializada para o desenvolvimento de quadros de servidores, gestores e agentes públicos que formulam e implementam políticas públicas. Ao longo do processo de formação da burocracia estatal no Brasil, a criação de escolas de governo assumiu um papel relevante para a formação de quadros de alto escalão, estruturação e profissionalização de carreiras de Estado e produção de mudanças culturais importantes para o aperfeiçoamento da administração pública.

A discussão sobre as escolas de governo e o seu papel na capacitação de servidores públicos surge com extrema importância, visto a necessidade constante de aprimoramento das políticas públicas e dos serviços prestados à população, sendo esta, uma das principais ferramentas para se atingir tais objetivos, refletindo-se em uma melhor geral da Administração Pública do país.

Sendo assim, o presente artigo tem como objetivo principal dissertar sobre o papel das escolas de governo no aperfeiçoamento e capacitação de servidores públicos.

Para alcançar o objetivo proposto, utilizou-se como recurso metodológico, a pesquisa bibliográfica, de natureza qualitativa e para fins descritivos. Para a obtenção dos textos e estudos científicos, realizou-se uma busca em bancos de dados digitais de publicações científicas, principalmente o Google Acadêmico e SciELO (Scientific Eletronic Library Online), utilizando-se dos descritores: escolas de governo; servidores públicos; capacitação; desenvolvimento.

O texto final foi fundamentado nas ideias e concepções de autores encontrados no levantamento bibliográfico, com destaque para as contribuições de: Andrade (2012), Pacheco (2002), Fernandes (2015) e Aires et al. (2014). 


\title{
RECIMA21 - REVISTA CIENTÍFICA MULTIDISCIPLINAR ISSN 2675-6218
}

ESCOLAS DE GOVERNO E SEU PAPEL NO APERFEICOAMENTO DO DESEMPENHO DOS SERVIDORES PÚBLICOS Adriano Mello de Andrade

\section{DESENVOLVIMENTO}

No decorrer dos anos, com as mudanças ocorridas nos meios sociais, políticos e econômicos, observou-se a necessidade de criar estratégias para auxiliar na capacitação dos servidores públicos, visando assim, o aperfeiçoamento das organizações públicas. De acordo com Pacheco (2002), a escola de governo trata-se de um termo indefinido, mas descrito de forma geral, se refere à uma instituição que se preocupa em preparar os funcionários públicos, buscando o desenvolvimento geral do mesmo, como profissional.

A capacitação dos profissionais do serviço público não se trata de uma ideia nova, já que, segundo pontua Andrade (2012, p. 24)

\begin{abstract}
Desde 1930, no Estado Novo, o governo Vargas defendia a profissionalização dos servidores como pressuposto para a construção de um governo forte, tendo sido criado, em 1938, o Departamento Administrativo do Serviço Público DASP. Entretanto, em virtude do domínio patrimonialista vigente na política brasileira, a expansão adequada de tal ideário não ocorreu a contento, criando apenas algumas ilhas de excelência.
\end{abstract}

Ou seja, o governo visualizava a importância de capacitar os servidores públicos de forma que demonstrasse o quanto o mesmo era um âmbito de força desde os tempos remotos, entretanto, somente a partir do século $X X$ foi que o tema de profissionalização foi revisto, a partir da percepção de que o desenvolvimento das habilidades dos servidores públicos era necessário, com o intuito de consolidar as instituições públicas e atingir o desenvolvimento da economia (ANDRADE, 2012).

Conforme apresenta Fernandes (2015, p. 4),

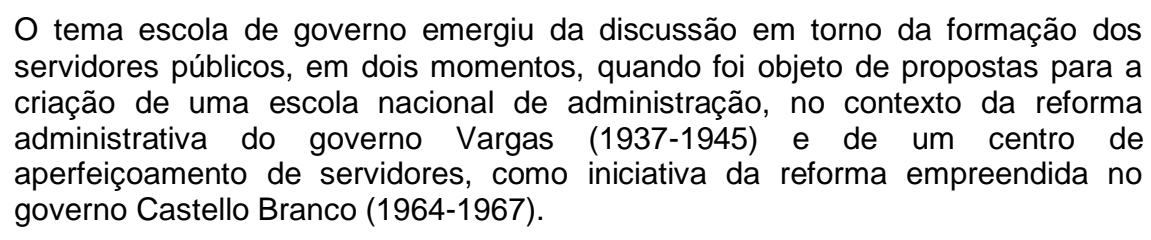

As escolas de governo foram organizadas através de decisões com e sem fins lucrativos, governamentais e não governamentais e acadêmicas e não acadêmicas. A partir do século XX, mais precisamente na última década, no Brasil, houve a multiplicação de diferentes modalidades de escolas de governo, por conta das opções instauradas na Reforma do Estado amparadas por propostas, ideologias políticas, ideológicas e teóricas (NOGUEIRA, 2005).

No Brasil, a escola de governo está prevista na Constituição Federal, que promulga, no § $2^{\circ}$ do artigo 39, a implantação e organização das mesmas em ambientes estaduais e federais, com o intuito de recepcionar as políticas de aperfeiçoamento e formação dos servidores públicos, proporcionando a qualidade do serviço público (BRASIL, 1988).

Para Aires et al. (2014, p. 1009), 


\title{
RECIMA21 - REVISTA CIENTÍFICA MULTIDISCIPLINAR ISSN 2675-6218
}

ESCOLAS DE GOVERNO E SEU PAPEL NO APERFEICOAMENTO DO DESEMPENHO DOS SERVIDORES PÚBLICOS Adriano Mello de Andrade

De acordo com o Decreto no 5.707, de 23 de fevereiro de 2006 (Brasil, 2006), as instituições destinadas, precipuamente, à formação e ao desenvolvimento de servidores públicos, incluídas na estrutura da administração pública federal direta, autárquica e fundacional, são consideradas escolas de governo. Esse decreto institui a Política Nacional de Desenvolvimento de Pessoal e explicita que as escolas de governo devem contribuir para a identificação das necessidades de capacitação dos órgãos e das entidades, considerando-as na programação de suas atividades.

Em relação aos tipos de instituições, há no Brasil, várias instituições relacionadas às diversas esferas governamentais, que se direcionam aos treinamentos, capacitações e também à formação profissional dos servidores públicos (ZOUAIN, 2003). Essas organizações podem ser privadas ou públicas, e segundo o autor, essas instituições podem ser classificadas em dois grupos:

\begin{abstract}
No primeiro, reúnem-se instituições que foram criadas com a missão de capacitar, em diversos níveis, os profissionais vinculados a áreas e carreiras específicas de atuação do Estado.

No outro grupo, estão as instituições que, não obstante atuarem com capacitação do setor público, não se vinculam, especificamente, à nenhuma carreira do Estado. São aquelas que têm sua atuação voltada para o grande contingente de servidores distribuídos em todos os níveis e áreas da burocracia do Estado (ZOUAIN, 2003, p. 1).
\end{abstract}

Com a infinidade de instituições existentes, percebe-se a multiplicidade das escolas de governo, que são direcionadas a formar servidores, capacita-los, aperfeiçoar as suas habilidades, formar cidadãos, dentre outras funções.

Zouain (2003), com o intuito de apresentar as diferenças entre escola de governo e centros de treinamento, esquematiza um quadro, no qual demonstra um perfil genérico para cada tipo de instituição.

Quadro 1 - Comparativo entre Escolas de Governo e Centros de Treinamento

\begin{tabular}{|l|l|l|}
\hline \multicolumn{1}{|c|}{ VARIÁVEL } & \multicolumn{1}{c|}{ ESCOLA DE GOVERNO } & \multicolumn{1}{c|}{ CENTRO DE TREINAMENTO } \\
\hline Público-alvo & Dirigentes Públicos* & Servidores Públicos \\
\hline Missão & Ampliar a capacidade de Governo & Aperfeiçoar a administração \\
\hline Objetivo & Formar líderes e dirigentes & Formar técnicos de carreiras \\
\hline $\begin{array}{l}\text { Áreas de conhecimentos } \\
\text { trabalhadas nos cursos }\end{array}$ & $\begin{array}{l}\text { Planejamento, gestão, processo } \\
\text { decisório, politicas públicas }\end{array}$ & $\begin{array}{l}\text { Técnicas de Administração, } \\
\text { Contabilidade, legislação, etc }\end{array}$ \\
\hline Demandas de cursos & Programas e Projetos de governo & Sistemas administrativos \\
\hline Resultados esperados & Eficácia das ações de Governo & Servidores atualizados \\
\hline
\end{tabular}

Fonte: Zouain (2003, p. 7)

A partir do comparativo, percebe-se que existem diferenças envolvendo o público-alvo, missões, objetivos, a área de conhecimento que são trabalhadas em cada instituição, demanda e resultados esperados, delimitando assim que as escolas de governo se preocupam em capacitar os profissionais de carreiras e áreas especificas que atuam no Estado. 


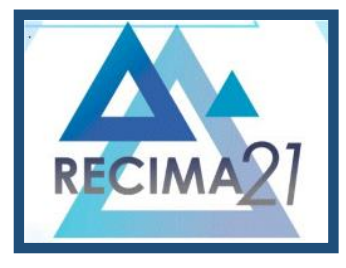

\section{RECIMA21 - REVISTA CIENTÍFICA MULTIDISCIPLINAR ISSN 2675-6218}

As escolas de governo possuem função de disseminar os conhecimentos em gestão pública, desenvolvendo as competências profissionais nos servidores públicos, visando um atendimento de qualidade (FERRAREZI; TOMACHESKI, 2010). As escolas de governo possuem diversas definições, sendo utilizadas para formar funcionários públicos, desenvolver fóruns de debates e reciclagem de servidores, principalmente aplicando-se nas três esferas de governo (federal, estadual e municipal) (BITTENCOURT; ZOUAIN, 2010).

Neste sentido, faz-se necessário evidenciar o papel das escolas de governo no aperfeiçoamento do desempenho dos servidores públicos. De acordo com Ranzini (2016), as escolas de governo surgem da necessidade de capacitação dos servidores públicos, no intuído de melhorar a qualidade dos serviços públicos prestados à população. Dessa maneira, é possível destacar a influência modernizadora da administração pública a partir da ação das escolas de governo, fomentando a capacitação de servidores públicos para a adoção de novos modelos de gestão pública.

As escolas de governo possuem papel central no desenvolvimento do funcionalismo públicos, fomentando um conhecimento teórico e prático que auxilia na tomada de decisões acerca das atividades da administração pública. Neste aspecto, é necessário que tais instituições têm papel fundamental no desenvolvimento profissional de servidores públicos, oferecendo conhecimentos essenciais para tornar as organizações públicas em órgãos ágeis e menor hierarquizadas, o que acarreta em uma melhoria da qualidade e eficiência dos serviços prestados por tais profissionais (BITTENCOURT; ZOAUIN, 2010).

Para Fernandes (2015), as escolas de governo possuem duas vertentes de atuação, que é a formação e quadros de carreiras e o da capacitação gerencial e treinamento coorporativo. $\mathrm{Na}$ primeira vertente, o objetivo é a formação de pessoas com vocação para ocupar cargos de assessoramento e direção. Já no caso da segunda vertente, o objetivo é a compatibilização do atendimento com as demandas apresentadas, promovendo um aperfeiçoamento gerencial e técnico dos servidores e, consequentemente dos órgãos onde desempenham as suas funções.

Segundo Ranzini (2016), as escolas de governo, por meio de programas de educação formal e de educação continuada, buscam realizar o aperfeiçoamento dos serviços prestados por servidores público, através de ações contínuas de capacitação, adequação dos perfis profissionais dos servidores que são requeridos pelo setor público e melhoria da eficácia e qualidade dos serviços prestados para o cidadão.

De acordo com Bittencourt e Zouain (2010), uma das pré-condições para o crescimento econômico de um país e a qualidade da Administração Pública e, para tanto, é necessário o seu desenvolvimento através das capacidades técnicas e gerenciais de seus servidores, criando um cenário favorável para a execução eficiente e produtiva de suas atividades. Nesse sentido, as escolas de governo são uma ferramenta para tal capacitação, por meio de programas de profissionalização dos servidores que visam aperfeiçoar as suas habilidades de gerência pública, 


\section{RECIMA21 - REVISTA CIENTÍFICA MULTIDISCIPLINAR ISSN 2675-6218}

ESCOLAS DE GOVERNO E SEU PAPEL NO APERFEICOAMENTO DO DESEMPENHO DOS SERVIDORES PÚBLICOS Adriano Mello de Andrade

instruir sobre a utilização e aplicação de tecnologia, entre outros aspectos que contribuem para a implementação eficaz das políticas públicas.

É possível afirmar que a formação dos servidores públicos é de extrema importância para o pleno funcionamento da máquina pública, bem como o a sua evolução perante as demandas sociais. Para que essas capacitações ocorram, são necessárias instituições com programas bem estruturados, contendo conteúdos pertinentes as funções exercidas por estes indivíduos. Assim, as escolas de governo surgem como uma estratégia de melhoria de desempenho das atividades governamentais (FERNANDES, 2015).

De maneira geral, é possível afirmar que as escolas de governo atuam na:

[...] a melhoria da qualidade dos serviços e a valorização dos servidores, através da readequação da política de recursos humanos, capacitação técnica e gerencial, estímulo à participação, melhoria nas condições de trabalho, criação de espaços de convivência dos servidores, redefinição do plano de carreira e avaliação de desempenho. A consolidação de uma máquina administrativa ágil e bem estruturada pleiteava estratégias de médio e longo prazo, valorizando a atuação de servidores e gestores eficientes, comprometidos e motivados (RANZINI, 2016, p. 90).

Para Aires et al. (2014), as escolas de governo possuem uma atuação significativa na vida do servidor público, qualificando-o, valorizando-o e construindo uma imagem renovada do serviço público. Tratam-se de instituições aptas para capacitar o funcionalismo público, constituindo-se como elementos de resistência contra técnicas administrativas ultrapassadas.

Sobre o assunto, Nascimento e Esper (2009) comentam que as escolas de governo possuem um papel de extrema importância, oferecendo programas de treinamento $\mathrm{e}$ desenvolvimento do funcionalismo público, bem como o seu reconhecimento como cidadão. Essas instituições contribuem de forma significativa para o aumento de desempenho nos órgãos públicos, ao oferecer ferramentas aos servidores para que possam se tornar mais capacitados.

Especificamente sobre a função das escolas de governo, Aires et al. (2014, p. 1010) argumentam que é a de:

[...] prospectar o que há de melhor em conhecimento e tecnologia gerencial, que provavelmente tenha se desenvolvido com antecedência no setor privado, e contextualizar este know-how às especificidades das organizações públicas. Deste modo, é essencial que esse tipo de instituição, visando tornar-se um centro de excelência, busque melhores práticas de gestão adotadas por outras escolas para identificar tendências e boas práticas em seu país e até mesmo no exterior.

Na visão de Carvalho, Santos e Fernandes (2014), o modelo de ensino propostos em escolas de governo visa a valorização e articulação dos saberes de servidores públicos, o que proporciona uma relação entre prática e teoria. Os autores destacam os principais desafios relacionados à Administração Pública são a complexidade das situações impostas aos servidores públicos e o caráter das demandas, crescentes e multifacetadas, já que os regimes democráticos 


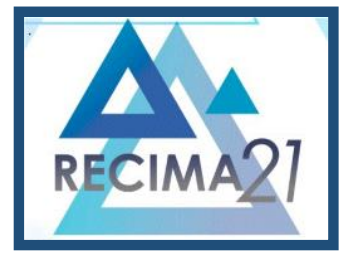

\section{RECIMA21 - REVISTA CIENTÍFICA MULTIDISCIPLINAR ISSN 2675-6218}

produzem uma expansão contínua de diretos, o que leva a uma necessidade de resposta do Estado.

Sob esse prisma, é possível dizer que cabem às escolas de governo dois papeis:

Por um lado, cabe fomentar debate e ajudar a construir modelo de gestão adequado ao Brasil, ajudando dirigentes na elaboração de soluções mais adequadas, algo estruturante e de longo prazo. Por outro lado, no curto prazo cabe capacitar servidores para exercer melhor suas funções, com qualidade e eficiência, pois a sociedade exige melhoria na qualidade dos serviços (CARVALHO; SANTOS; FERNANDES, 2014, p. 16).

Tratando sobre a atuação das escolas de governo, Fernandes (2015) destaca a formação e capacitação de servidores públicos com perfil de liderança, capazes de resolver problemas de maneira autônoma e eficaz, trazendo maior agilidade para os processos.

A partir dos pontos destacados no presente artigo, fica evidente o papel transformador das escolas de governo na Administração Pública e a sua influência no aperfeiçoamento do desempenho de servidores públicos, agindo de maneira a agregar com as suas atuações e, dessa maneira, melhorando a qualidade dos serviços prestados.

\section{CONCLUSÃO}

A pesquisa em questão buscou apresentar sobre a escola de governo e o seu papel no aperfeiçoamento do desempenho dos servidores públicos, delimitando informações objetivas sobre o assunto, conhecimentos esses que auxiliassem na construção do trabalho, com a intenção de esclarecer pontos relevantes acerca do surgimento das escolas de trabalho e sua função.

A sociedade exige do governo atendimentos públicos cada vez mais excelentes e nesse sentido acabam exigindo maior transparência e qualidade da Administração Pública. Nesse contexto, as escolas de governo possuem papel essencial na capacitação profissional dos servidores, comprometidos com uma nova postura adotada pelo Estado, preocupando-se com uma Administração Pública de qualidade.

$\mathrm{Na}$ pesquisa, elencou-se o surgimento das escolas de governo, as suas classificações e como funcionam as suas ações com o intuito de aperfeiçoar o desempenho dos servidores públicos, prezando pela formação crítica dos mesmos, estimulando-os a serem proativos e responsáveis pelo desenvolvimento de suas habilidades.

Em conclusão, é preciso que os gestores governamentais estejam conscientes sobre a importância de estabelecer a capacitação continuada aos servidores públicos, prezando pelo pleno desenvolvimento dos funcionários e auxiliando na formação de sujeitos críticos, autônomos e participantes ativos do projeto político da sociedade, estimando pela cidadania e colaborando para uma Administração Pública eficaz e transparente, atendendo assim, as expectativas de todos os membros da sociedade. 


\section{RECIMA21 - REVISTA CIENTÍFICA MULTIDISCIPLINAR ISSN 2675-6218}

ESCOLAS DE GOVERNO E SEU PAPEL NO APERFEICOAMENTO DO DESEMPENHO DOS SERVIDORES PÚBLICOS Adriano Mello de Andrade

\section{REFERÊNCIAS}

AIRES, Renan Felinto de Farias et al. Escolas de governo: o panorama brasileiro. Revista de Administração Pública, v. 48, n. 4, p. 1007-1027, 2014. Disponível em: https://www.scielo.br/scielo.php?pid=S0034-76122014000400010\&script=sci arttext. Acesso em: 28 set. 2020.

ANDRADE, Patrícia Roberta Paes de. Educação profissional na administração pública: desenvolvendo competências para atender às demandas da sociedade brasileira. 2012. $209 \mathrm{f}$. Tese (Doutorado) - Fundação Getúlio Vargas, Rio de Janeiro, 2012. Disponível em: https://bibliotecadigital.fgv.br/dspace/bitstream/handle/10438/10555/Dissertacao mestrado Patrici a Andrade versao final completa\%5B1\%5D.pdf. Acesso em: 27 set. 2020.

BITTENCOURT, Maryângela Aguiar; ZOUAIN, Deborah Moraes; Escolas de governo e a profissionalização do Servidor Público: estudo dos casos da Escola de Serviço Público do Estado do Amazonas-ESPEA e da Fundação Escola de Serviço Público Municipal de ManausFESPM. Revista Adm. Made, v. 14, n. 2, p. 75-94, 2010. Disponível em: http://revistaadmmade.estacio.br/index.php/admmade/article/view/83. Acesso em: 28 set. 2020.

BRASIL. Constituição da República Federativa do Brasil de 1988. Brasília: Senado Federal, 1988. Disponível em: http://www.planalto.gov.br/ccivil 03/constituicao/constituicao.htm. Acesso em: 27 set. 2020.

CARVALHO, Paulo Sergio de; SANTOS, Handemba Mutana Poli dos; FERNANDES, Ciro Campos Christo. Trajetória e perspectivas de uma escola de governo: a experiência da Enap na administração federal. VII Congresso de Gestão Pública - CONSAD, 2014. Disponível em: https://repositorio.enap.gov.br/handle/1/1751. Acesso em: 28 set. 2020.

FERNANDES, Ciro Campos Christo. Escolas de governo: Conceito, origens, tendências e perspectivas para sua institucionalização no Brasil. VIII Congresso de Gestão Pública CONSAD, 2015. Disponível em: https://repositorio.enap.gov.br/handle/1/2238. Acesso em: 27 set. 2020.

FERRAREZI, Elisabete; TOMACHESKI, João A. Mapeamento da oferta de capacitação nas escolas de governo no Brasil: gestão da informação para fortalecimento da gestão pública. Revista do Serviço Público, Brasília: ENAP, v. 61, n. 3. p. 287-303, 2010.

FONSECA, Diogo Ribeiro da et al. Sistema de Escolas de Governo da União: perfil, identidade e desafios para institucionalização. Brasília: Enap, 2015. 137 p. Cadernos ENAP.

NASCIMENTO, Tarcilena P. C.; ESPER, Aniely K. Evasão em cursos de educação continuada a distância: um estudo na Escola Nacional de Administração Pública. Revista do Serviço Público, v. 60 , n. 2 , p. $159-173,2009$.

NOGUEIRA, Marco Aurélio. Atualidade, força e sentido das Escolas de Governo. In.: Um Estado para a sociedade civil: temas éticos e políticos da gestão democrática. São Paulo: Cortez, 2005. p. 167-193.

PACHECO, Regina Silva. Escolas de Governo como centros de excelência em gestão pública: a perspectiva da ENAP - Brasil. Revista do Serviço Público. Brasília: v. 53, n. 1, jan./abr. 2002.

RANZINI, Milena de Senne et al. Escolas de governo municipais paulistas: estudo de casos dos municípios de Guarulhos, São Paulo e Sorocaba. 2016. 190 f. Dissertação (Mestrado) - 


\section{RECIMA21 - REVISTA CIENTÍFICA MULTIDISCIPLINAR ISSN 2675-6218}

ESCOLAS DE GOVERNO E SEU PAPEL NO APERFEICOAMENTO DO DESEMPENHO DOS SERVIDORES PÚBLICOS

Universidade Estadual de Campinas, Campinas, 2016. Disponível em: http://repositorio.unicamp.br/handle/REPOSIP/305313. Acesso em: 28 set. 2020.

ZOUAIN, Deborah Moraes. Escolas de governo e escolas de serviço público: limites e problemas. Estudo de caso da FESP/RJ e FUNDAP/SP. VIII Congresso Internacional del CLAD sobre la Reforma del Estado y de la Administración Pública. Panamá: 2003. 